\title{
Microarray spot detection using flat structuring periodic line and specific distance shaped morphology
}

\author{
Kadam A.B., Manza R.R. and Kale K.V.* \\ ${ }^{*}$ Department of Computer Science and Information Technology, Dr. Babasaheb Ambedkar Marathwada University, \\ Aurangabad. (MS) India, E-mail: avinashkadam123@rediffmail.com
}

\begin{abstract}
Microarray is one of the most recent and important technology for exploring the genome [1,2]. Image analysis is an important role of microarray experiments, one, which can have a potentially large impact on subsequent analysis such as clustering or the identification of differentially expressed genes [3]. Microarray image grid and spot position determination is a very important step in the analysis of microarray image because it is the first part we need to do for the analysis. By comparing gene expression in normal and abnormal cells, microarrays may be used to identify genes, which are involved in particular diseases. These genes may then be targeted by therapeutic drug. Making this part automated and fast is also important. So we consider this as indeed problem of spot detection and overcome it by using flat structuring element disk-diamond shaped morphological technique, which gives satisfied results. And also we compare the original \&resultant image results on the basis of image quality measure [4].
\end{abstract}

Keywords- Microarray, flat structuring element, morphology, segmentation, spot detection

\section{Introduction:}

The Analysis of microarray gene expression data involves many steps, the initial step consist of extracting the gene expression data from the microarray image, and include spot localization or gridding, foreground and background separation, intensity extraction [3]. These stages are quite important, since accuracy of the resulting data is essential in posterior analysis [5]. The DNA sequences are spotted on the slides. After image processing, gene expression data can be obtained for further analysis, such as gene clustering, identification [6], segmentation- fixed circle, adaptive circle, adaptive shape, histogram, and Seed region growing, addressing, information extraction, adaptive ellipse method $[3,7]$. However, one of the major challenges of this approach is the image-processing phase. The purpose of this step is to extract each spotted

DNA sequence as well as to obtain background estimates and quality measures [6]. DNA microarray can also be used to characterize the cellular differences between different responses and between different tissue types, such as between normal cells and cancer cells, or between cancers with different responses to treatment, or between control cells and cells treated with a particular drug. [6]

Motivation from this problem, in this paper we have applied morphological segmentation technique to determine these spots and got satisfied result as well. And compare disk and diamond shaped structuring element and calculate the statistical parameters of original and resultant image our results are visibly and statistically more good and clear.

\section{Microarray Image Segmentation:}

Segmentation and background correction methods are used for extraction of information from microarray. In some cases background adjustment can substantially reduce the precision that is increase the variability of low intensity spot values [9]. The background estimation approach uses an image analysis technique. Out of the three steps of Microarray image processing, Segmentation allows the classification of pixels either as foreground that is corresponding to a spot of interest or as background. In reported work existing segmentation techniques are $[3,4$, \& 6]:
1. Fixed circle segmentation
2. Adaptive circle segmentation
3. Adaptive shape segmentation
4. Histogram segmentation
5. Adaptive ellipse method.

But every method or technique have its own advantages and disadvantages, as mentioned in recent work fixed circle segmentation is easy to implement but a fixed diameter segmentation is clearly not satisfactory for all spots this technique is implemented by M. B. Esien software name is ScanAlyze [3]. In adaptive circle segmentation circles diameter is estimated separately for each spot but this can be time consuming since each array contain thousand of spots this technique is implemented by M. B. Esien software name is ScanAlyze \& GenePix. Where as in adaptive shape segmentation two methods are used watershed and seed region growing. Both watershed \& SRG requires the specification of starting point of segmentation procedures using these methods can be the selection of the number \& location of the seed points. Microarray images are well suited to such techniques. But in histogram segmentation method for each spot foreground and background intensity estimates 
are determined on the basis of histogram of pixel values [3]. These methods do not use any local spatial information and these are simple methods. The main disadvantage of this method is that quantitation is unstable when a large target mask is set to compensate for spot size. These methods exist for microarray segmentation but no one method produces the increase in number of spots, which are present in the image after segmentation of background and foreground. To overcome this we have set the objective for this paper and overcome this problem up to certain level by using morphological transforms with some morphological operations.

\section{Methodology:}

Structuring element (SE), of the type specified by shape. Depending on shape, structuring element can take additional parameters. There are two types of structuring element [10]:

i) Flat Structuring Element, ii) Non-flat Structuring Element

Basically, Flat structuring element are used for two dimensional image and Non flat structuring element for three-dimensional object, the parameter added for Non-flat i.e. height. The structuring element like: Disk, Diamond, Arbitrary, Pair, Periodic line, Rectangle, Line, Disk and Octagon are belongs to flat structuring element, whereas Arbitrary and Ball are the non-flat Structuring element.

\section{Morphological Operations:}

Morphology technique is used to operate on images via different operations; few of them are given here.

\section{Closing and Opening:}

By iteratively applying erosion and dilation, one can eliminate image details, smaller than the structuring element, without affecting its global geometric features.

Visually, closing smoothes the contours by filling in narrow gulfs and eliminating small holes. The closing operation is simply a dilation operation followed by an erosion operation. The gray scale closing of $\mathrm{A}$ by $\mathrm{B}$, denoted by $(A \bullet B)$, is defined as,

$$
(A \bullet B)=(A \oplus B) \Theta B
$$

On the other hand, opening smoothes contours by breaking narrow isthmuses and suppressing small islands. The opening operation is simply an erosion operation followed by a dilation operation. The gray scale opening of $A$ by $B$, denoted by $(A \circ B)$, is defined as,

$$
(A \circ B)=(A \Theta B) \oplus B
$$

In this section we briefly introduce the basic principles, definitions and notations used in mathematical morphology $[9,11]$.

When we perform the morphological operation like dilation and erosion the structuring element plays an important role, which is a powerful tool to extract information from images [4]. Structuring element is a small grid representing pixels. Where as erosion and dilation are considered the primary morphological operations and the operations of opening and closing are secondary operations and these are implemented using erosion and dilation operations. When we subtract the opened image from the original image is known as Top-hat Transform $\left(T_{h}\right)$, which is defined as,

$$
T_{h}=I-(I \circ B)
$$

When we subtract the closed image from the original image $(\mathrm{I})$ is known as Bottom-hat

Transform ( $\left.{ }^{B_{h}}\right)$ and which is defined as,

$$
B_{h}=I-(I \bullet B)
$$

Where, $I$ is the image, $B$ is the Structuring element.

In this proposed work first, we read the original grayscale image then do start further analysis. In next step add this image to the top hat transform, and then subtract the bottom-hat transform i.e. we perform bottom hat \& tophat operation on input image by using disk, diamond shaped structuring element. Then adjust intensity of the image and repeat both procedures. After that, we extract the background of the image by using morphological opening operation having disk \& diamond shaped structuring element. Here we have used structuring element, whose radius changes the radius (up to 1 - 9) that found good to get satisfied result and then subtract the background from the adjusted image and get final result, which are satisfied and promising. But these all combinations of disk-disk, disk-diamond, diamond-diamond, diamond-disk of structuring elements are different types of statistical results are found. As this paper deals with morphological segmentation technique for various image segmentation mathematical morphology, which is very attractive because it efficiently deals with geometrical features such as size, shape [4, 12], contrast, or connectivity that can be considered as segmentation oriented features. The basic features of structuring elements are, Disk specifies periodic-line $S . E$., radius ' $R$ ' must be non-negative integer \& Diamond specify the distance from the $S . E$., radius 'R' must be nonnegative integer scalar.

Mean:

$m=\sum_{i=0}^{L=1} z_{i} p\left(z_{i}\right)$ 
Standard Deviation:

$$
\sigma=\sqrt{\mu_{2}(z)}=\sqrt{\sigma^{2}}
$$

Mean is to measure a average intensity and Standard deviation is to measure average contrast. Where, $z_{i}$ is a random variable indicating intensity, $p(z)$ is the histogram of the intensity levels in a region, $L$ is the number of possible intensity levels.

Proposed Algorithm: as shown in fig. (1)

\section{Result and Discussion:}

In this paper, we have applied new morphological technique to determine the spot by segmenting the background and noisy region of the image, which gets spot identification easy as well as we can easily observe the increase in number of spot. For this experiment we have taken microarray images and worked on it, out of that the fig. $\mathbf{2}$ is the original image, which is the problem in microarray, and fig. $3 \& 4$ is the resultant microarray images. We have compared the spots before and after applied the proposed technique respectively. As mention the mathematical morphology techniques are useful for microarray segmentation but in this spot identification process structuring element of shapes and radius is a change at various times and we seen various types of results visually and statistically as shown in table (1) \& graph (1) shows Mean \& STD Vs Shape \& radius. Also we analyze number of 8 combinations of S. E. \& 80 different microarray images. Generally microarray images is contains number of spots is circular but we use two types of structuring elements shapes such as disk, diamond \& also to change the radius up to 1 to 9 as shown in table (1). Firstly we check the shape is disk-disk and radius is 2-1, 2$2, \ldots, 2-9$, etc. \& then change shapes diskdiamond, diamond-diamond, diamond-disk, as like this. It is also found that visibility of the image and clarity is better. And statistically the calculated mean and STD before and after is also good than other combination, because by using morphology it separate foreground \& background but spots are could not clearly separated. In addition we have check one additional parameter that is new one (object count), by using this the number of spots is increased as compare to manual count; because of morphology objects are clearly separated from each other, in this case it is considered to the false rejection rate.

\section{Conclusion \& Future Work:}

In this paper, we have given the results of morphological segmentation technique on microarray image for detecting the spot; it improves the number of gene position determination, as shown in the results these techniques work well for microarray spot detection than the other existing techniques such as fixed circle segmentation, \& watershed, etc. In this experiment we use these two shapes of eight combinations with different 80 images but overall performance is best by using disk-disk or diamond-disk \& having radius $2-9$. In future work, we try to improve the present drawbacks $\&$ check the other combinations of structuring elements such as square \& it adds with diamond, disk shapes \& also observe the changes between these all combinations and finally, which is a best method is used to produce an automated, fast and simple gene identification system.

\section{References:}

[1] Basim Alhadidi, Hussam Nawwaf Fakhouri, and Omar S. AlMousa, "cDNA Microarray Genome Image Processing Using Fixed Spot Position", American Journal of Applied Science 3(2): 17301734, 2006.

[2] Yee Hwa Yang, Michael J. Buckley, et.al, "Comparison of methods for image analysis on cDNA microarray data", Department of statistics, University of California at Berkeley, Technical report vpl 584, Nov, 2000.

[3] Rafael C. Gonzalez, Richard E. Woods, Digital Image Processing,, Pearson Education, 2004.

[4] Luis Rueda \& Li Quin, "An unsupervised Learning Schme for DNA microarray image spot detection",http://citeseerx.ist.psu.edu/viewdoc/summ ary? doi=10.1.1.59.7982

[5] Robert S. H. Istepanian, "Microarray Image Processing: Current Status and Future Directions", IEEE Transaction on Nanobioscience, Vol.2 No.4Dec-2003.

[6] Luis Rueda \& Li Quin, "A new method for DNA microarray image segmentation", http://citeseerx.ist.psu.edu/viewdoc/summary? doi=10.1.1.59.8958

[7] Peter Bajcsy, Lei Liu and Mark Band, "DNA Microarray Image Processing", reference link=>isda.ncsa.uiuc.edu/peter/publications/.../draftD NAPressChapter_v8.pdf

[8] A. Bengtsson, "Microarray Image Analysis: Background Estimation Using Region and Filtering Techniques", Master's theses, Lund University, vol. E40, 2003.

[9] Kasif I. Siddiqui, Alfred O. Hero, \& Matheen M. Siddiqui, "Mathematical Morphology applied to spot segmentation and quantification of gene microarray images", Reference Link=> www.eecs.umich.edu/ hero/Preprints/kashif_asilom ar2002.pdf

[10] www.mathswork.com

[11] Edward R. Dougherty, Roberto A. Lotufo, Hands-on Morphological Image Processing, (tutorial texts in optical Engineering volume TT59, SPIE Press) 2003.

[12] Mohsen Abbaspur, Rafeef Abugharbieh, et.al. " Hybrid spot segmentation in four channel microarray genotyping image data", IEEE International Symposium on Signal Processing and Information Technology, page no. 11-16, Aug-2006. 


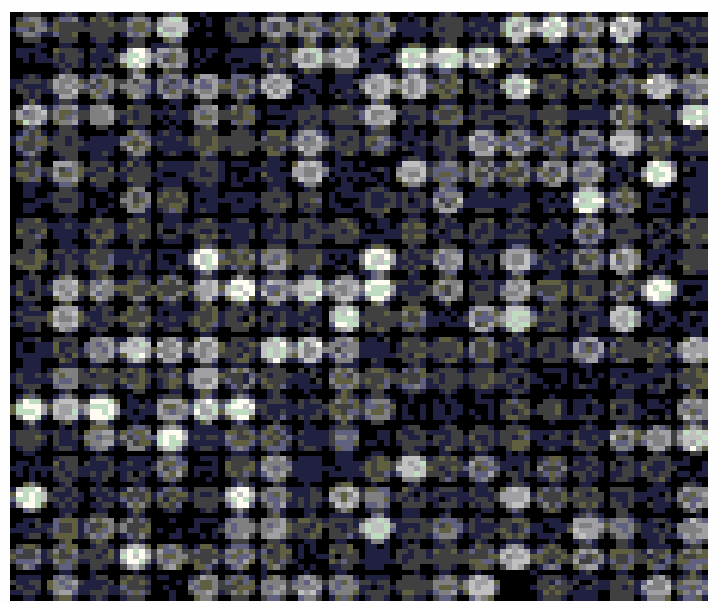

Fig.2- Original image

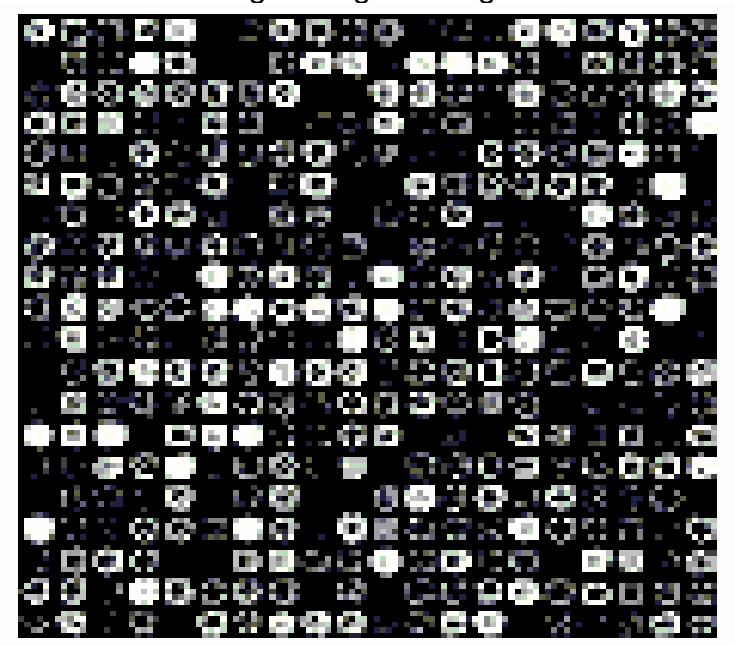

Fig.3-Resultant Image disk-disk [2,9]

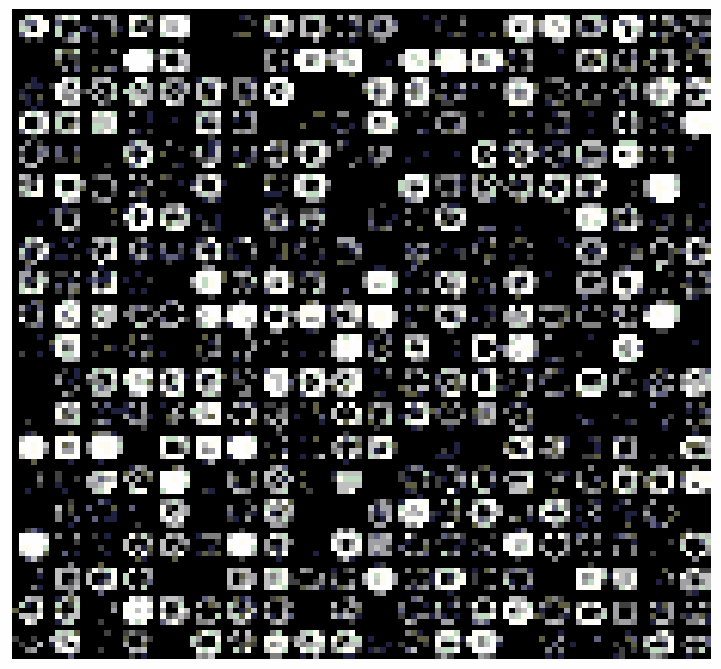

Fig.4-diamond-disk [2,9] 
Table: 1: Result of microarray image (original \& Resultant) using Two shapes Disk \& Diamond

\begin{tabular}{|c|c|c|c|c|c|c|c|}
\hline $\begin{array}{c}\text { Combination } \\
\text { of S.E. }\end{array}$ & Radius & \multicolumn{3}{|c|}{ Original Image } & \multicolumn{3}{c|}{ Resultant image } \\
\cline { 2 - 8 } & & Mean & STD & No(object) & Mean1 & STD1 & No(object) \\
\hline Disk-Disk & 2,9 & 62.0736 & 52.7239 & 369 & 55.7637 & 87.0535 & 683 \\
\cline { 2 - 8 } & 9,2 & 62.0736 & 52.7239 & 369 & 70.2414 & 100.6111 & 594 \\
\hline \multirow{2}{*}{$\begin{array}{c}\text { Diamond- } \\
\text { Diamond }\end{array}$} & 2,9 & 62.0736 & 52.7239 & 369 & 59.4894 & 89.5566 & 689 \\
\cline { 2 - 8 } & 9,2 & 62.0736 & 52.7239 & 369 & 73.6939 & 101.8939 & 593 \\
\hline $\begin{array}{c}\text { Disk- } \\
\text { Diamond }\end{array}$ & 2,9 & 62.0736 & 52.7239 & 369 & 59.4894 & 89.5566 & 689 \\
\cline { 2 - 8 } & 9,2 & 62.0736 & 52.7239 & 369 & 70.2414 & 100.6111 & 594 \\
\hline $\begin{array}{c}\text { Diamond- } \\
\text { Disk }\end{array}$ & 2,9 & 62.0736 & 52.7239 & 369 & 55.7637 & 87.0535 & 683 \\
\cline { 2 - 8 } & 9,2 & 62.0736 & 52.7239 & 369 & 73.6939 & 101.8939 & 593 \\
\hline
\end{tabular}

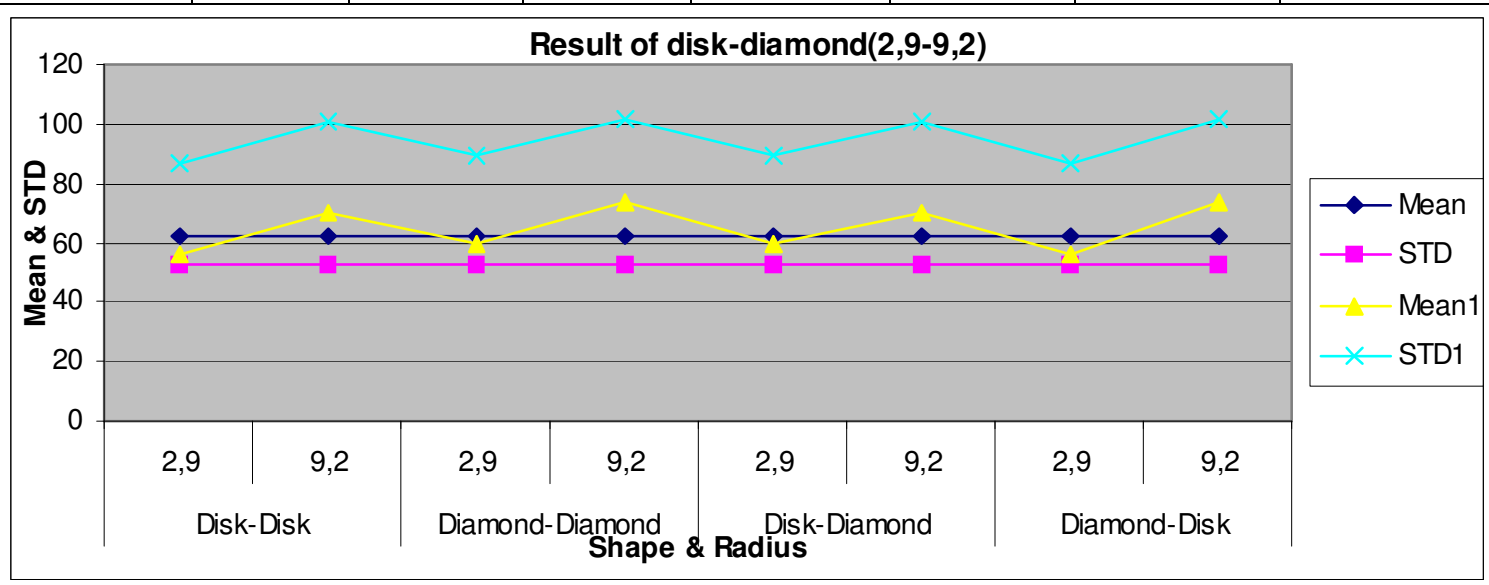

Graph (1): Graph shows x-axis contains Shapes \& Radius and y-axis contains Mean \& STD

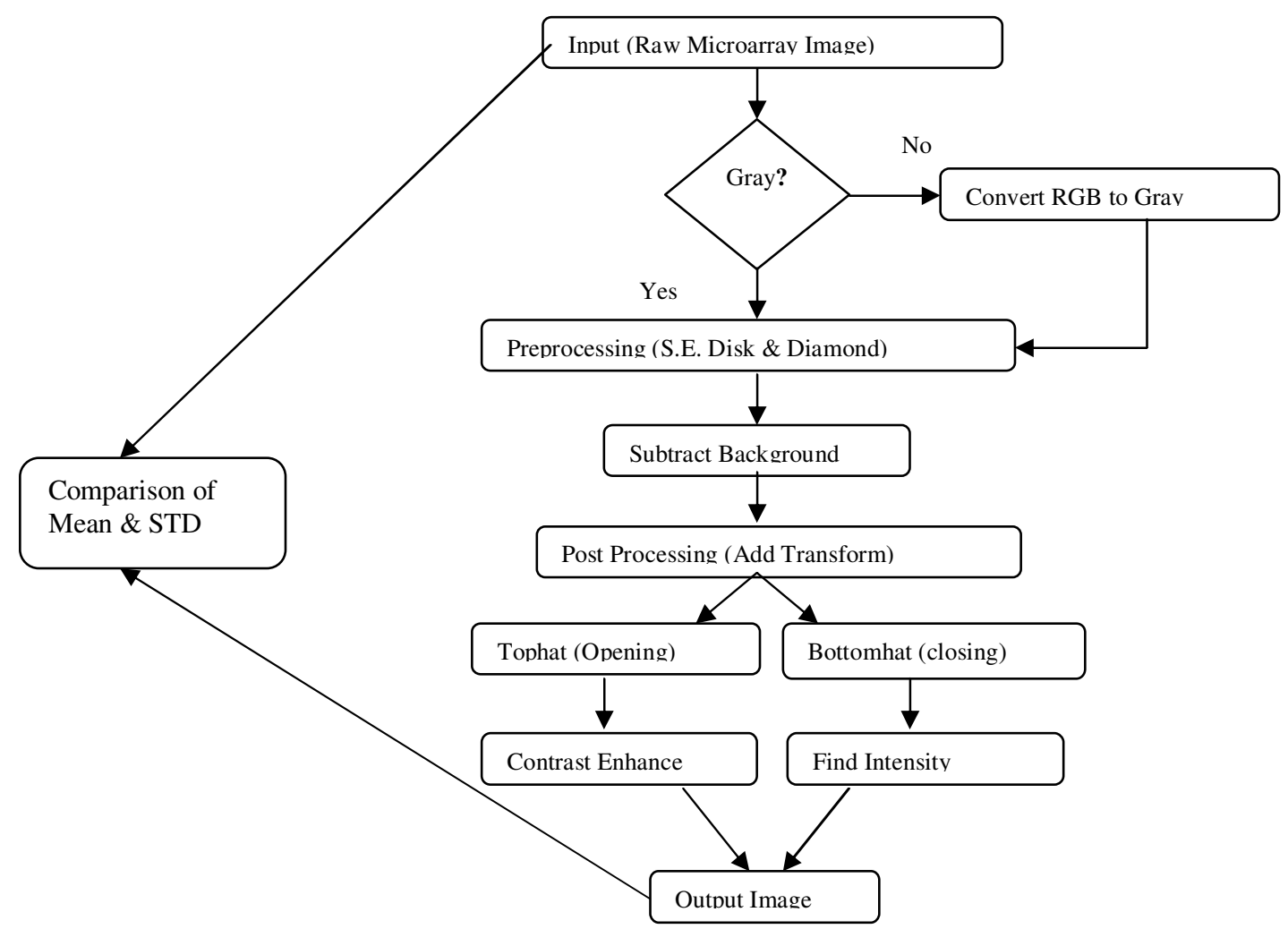

Figure1. Proposed Algorithm for Morphological Microarray Image segmentation 